\title{
Wide band-gap oxide nanoparticles as potential drug carriers ${ }^{1)}$
}

\author{
WALDEMAR LIPIŃSKI, JAROSŁAW KASZEWSKI*, **, ZDZISŁAW GAJEWSKI, \\ MAREK GODLEWSKI**, MICHAt M. GODLEWSKI*
}

\author{
Department of Large Animal Diseases with Clinic, Faculty of Veterinary Medicine, \\ Warsaw University of Life Sciences - SGGW, Nowoursynowska 100, 02-797 Warsaw, Poland \\ *Department of Physiological Sciences, Faculty of Veterinary Medicine, \\ Warsaw University of Life Sciences - SGGW, Nowoursynowska 159, 02-776 Warsaw, Poland \\ **Institute of Physics, Polish Academy of Sciences, Al. Lotników 32/46, 02-668 Warsaw, Poland
}

\section{Lipiński W., Kaszewski J., Gajewski Z., Godlewski M., Godlewski M. M. Wide band-gap oxide nanoparticles as potential drug carriers}

\section{Summary}

Recent decades have brought rapid development in the field of nanotechnology, which has led to applications of nanoparticles in many industries. Unique properties of nanoparticles and their biocompatibility increase their potential as drug carriers in drug-delivery systems. Prof. Marek Godlewski's team from the Institute of Physics PAS has developed wide band-gap metal oxide nanoparticles doped with rare-earth metals for applications as fluorescent markers. The potential of those nanoparticles to cross the closed gut barrier after alimentary application has prompted their use in drug delivery systems. In this study, we show that after conjugation with a model bioactive substance, lectin (Phaseolus vulgaris), these nanoparticles retained their advantageous properties and, following oral administration $(10 \mathrm{mg} / \mathrm{ml}$ in $\mathrm{RO}, 0.3 \mathrm{ml} / \mathrm{mouse})$, entered a variety of organs in the mouse model. Internal organs collected at key time points were analysed under a scanning cytometer and a confocal microscope. The results show that the conjugation reduced, but did not completely abolish, the capacity of nanoparticles to penetrate physiological barriers (intestinal, blood-brain barrier) in the organism.

Keywords: nanoparticles, drug delivery system, lectin

Over the years, nanoparticles (NPs) have found numerous applications in various industries thanks to their unique properties (8). In the field of biomedicine, one of the most researched applications are drug delivery systems (DDS) $(7,8)$. Many NPs exhibit properties advantageous for DDS, such as the ease of modification, low toxicity, ability to penetrate physiological barriers, favourable biodistribution, biocompatibility and biodegradability $(3,5,9-15)$. Furthermore, NPs may also protect a drug from degradation and increase its biological stability, bioavailability and retention time. Various materials have been used as NPs (proteins, lipids, metals, metal oxides, nucleic acids) for DDS, each with its own advantages and unsolved problems $(9,10,14,15)$.

1) The research was partially funded by grants from the National Science Centre: DEC-2012/05/E/NZ4/02994 and DEC-2012/06/A/ST7/00398. This work was supported by the projects Veterinary Research Centre and Biomedical Research Centre conducted at the Warsaw University of Life Sciences.
Prof. Marek Godlewski's team from the Institute of Physics, Polish Academy of Sciences (IP PAS), have developed NPs based on wide band-gap semiconductor metal oxides doped with rare earth metal ions to activate their fluorescence $(3,5,11-13)$. These NPs, in addition to their strong fluorescence, exhibit many properties important for DDS. Their surface can be easily modified because it is terminated by hydroxide groups. They exhibited low toxicity $(2,5)$, could penetrate physiological barriers, including intestinal and blood-brain barrier and were widely distributed within the body $(3,5,11-13)$. In addition, these NPs were either biocompatible or biodegradable (5).

The goal of this study was to determine whether orally administered wide band-gap metal oxide NPs conjugated with a model bioactive substance retain their ability to penetrate through physiological barriers within the body. For the purpose of our experiments, we utilised $\mathrm{Y}_{2} \mathrm{O}_{3}: \mathrm{Tb}$ NPs (red fluorescence) conjugated 
with lectin from Phaseolus vulgaris, a substance that normally does not cross the closed gut barrier.

\section{Material and methods}

If not mentioned otherwise, all chemicals were purchased from Sigma-Aldrich (Poland)

$\mathrm{Y}_{2} \mathrm{O}_{3}$ : Tb nanoparticles preparation and conjugation with lectin. Yttrium oxide NPs were prepared in IP PAS by the hydrothermal method, as described in Kaszewski et al (4). Powdered lectin was kindly provided by Biolek (Poland). Composite of yttrium oxide-lectin was synthesized as follows. Yttrium oxide NPs were calcined at $1200^{\circ} \mathrm{C}$ in the air atmosphere for $2 \mathrm{~h}$. After a cooling process, $0.3 \mathrm{~g}$ of nanopowder and $0.3 \mathrm{~g}$ of lectin were added to $30 \mathrm{ml}$ of deionized water. Suspension was prepared using a Vibra Cell VCX500 (Sonics) ultrasonic processor. Sonication was performed at $80 \%$ of power with 12 cycles of 1 minute pulse on and 1 minute pulse off. The resulting homogenous suspension was then hotplated at $80^{\circ} \mathrm{C}$ to evaporate all the water. The dry composite was then ground in an agate mortar and stored in a PP vial. Prior to application, NPs were resuspended in RO water $(10 \mathrm{mg} / \mathrm{ml})$ and sonicated to obtain a homogenous suspension.

Mice experiments. Healthy adult Balb-c mice (3 months and older) purchased from IMDiK PAN were used for the experiment. All procedures were approved by the Local Ethical Committee (approval No. 44/2012) and conducted according to EU and local directives. Before any experimental procedures, mice went through a 7-day acclimation period. During the experiment, the mice were kept in individual cages under standard living conditions, fed $a d$ libitum with standard sustenance feed with unlimited access to water. For the NPs gavage procedure, mice were immobilised, and $0.3 \mathrm{ml}$ of the suspension of NPs in RO water was administered by gastric gavage by means of a Hauptner Herberholz 31011 needle (Mezaret S.A., Poland) with a modified profile. After $24 \mathrm{~h}, 48 \mathrm{~h}$ and 1 week, the mice were sacrificed, and tissues were collected for analysis.

For the purpose of the experiment, the following tissues were collected: duodenum, liver, kidneys, spleen and brain. The samples were embedded in paraffin, cut into $5 \mu \mathrm{m}$-thick sections and mounted on microscope slides. After a rehydration procedure, the samples went through an antigen restoration process $\left(90^{\circ} \mathrm{C}\right.$ microwave in citric buffer) prior to labelling. The following reagents were used: anti-lectin antibodies (1:100 in PBS, $4^{\circ} \mathrm{C}$, over-night) and secondary Alexa Fluor 488-conjugated antibodies (1:250 in PBS, room temperature, $1 \mathrm{~h}$ ). Additionally, the samples were counterstained with Hoechst $33342(1 \mathrm{mg} / \mathrm{ml}$, room temperature, $1 \mathrm{~min})$. Afterwards, the samples were covered with mounting medium for immune-fluorescence and sealed under coverslips.

The labelled samples were evaluated under a scanning cytometer $\mathrm{SCAN}^{\wedge} \mathrm{R}$ (Olympus, Poland) and a confocal microscope Leica SP8-WLL (KAWA.SKA, Poland). The following excitation-emission ranges were used: $405 \mathrm{~nm}$ vs. $420-450 \mathrm{~nm}$ for HOECHST
$33342,488 \mathrm{~nm}$ vs. $505-525 \mathrm{~nm}$ for Alexa Fluor 488 and $610 \mathrm{~nm}$ IF vs. $625-650 \mathrm{~nm}$ for $\mathrm{Y}_{2} \mathrm{O}_{3}: \mathrm{Tb}$ NPs.

Statistical evaluation. The data obtained were expressed as mean values \pm SEM. For statistical evaluation of $\mathrm{SCAN}^{\wedge} \mathrm{R}$ experiments, a one-way ANOVA with a TukeyKramer multiple comparison test were applied, using GraphPad InStat 3.10. For all tests, significance was set at $95 \%$ or $99 \%$ confidence level with $\mathrm{p} \leq 0.05$ treated as significant, and $p \leq 0.01$ and $p \leq 0.001$ as highly significant.

\section{Results and discussion}

Scanning cytometry and confocal microscopy reveal the extent of the persorption of $\mathrm{Y}_{2} \mathrm{O}_{3}:$ Tb-Lectin complexes in the duodenum.

Twenty-four hours after IG, a high percentage of cells were lectin positive (Fig. 1), with lectin found both inside enterocytes and in the brush border region (Fig. 2, arrows). Although the percentage of lectin-positive cells increases over time (Fig. 1) with statistical significance between $24 \mathrm{~h}$ and 1 week, the amount of lectin associated with the brush border diminished over time (Fig. 2, red arrows, compare $24 \mathrm{~h}$ with $48 \mathrm{~h}$ ). Furthermore, in all time points post IG, the index of cells positive for NP-lectin complexes remained unchanged (Fig. 1), while the percentage of cells positive for free NPs increased over time (Fig. 1) with statistical significance between $24 \mathrm{~h}$ and 1 week.

Distribution of $\mathrm{Y}_{2} \mathrm{O}_{3}$ : Tb-Lectin complexes in the liver and kidney. In the kidney, the results showed

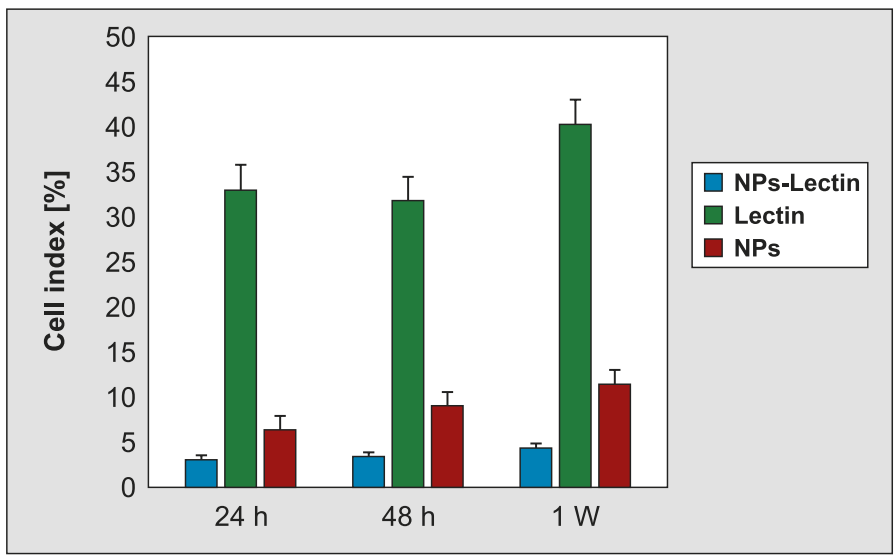

Fig. 1. Average index ( \pm SEM) of cells positive for NP-Lectin complexes, lectin and NPs alone at $24 \mathrm{~h}, 48 \mathrm{~h}$ and 1 week (1 W) post IG in the duodenum
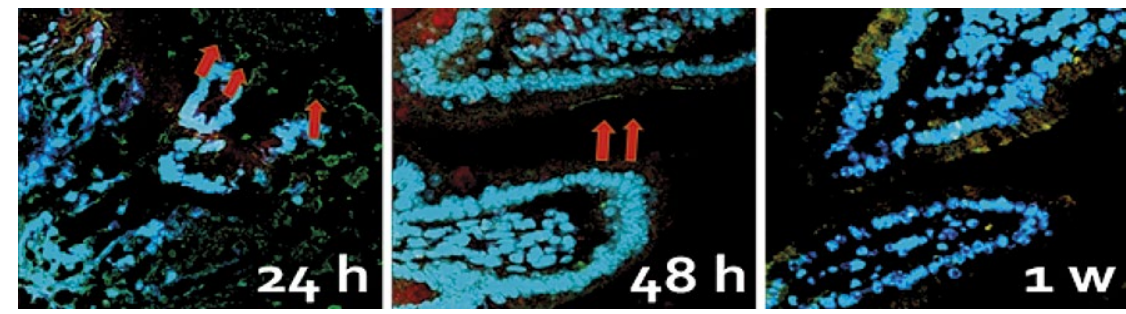

Fig. 2. Confocal image $(20 \times)$ from the duodenum at $24 \mathrm{~h}, 48 \mathrm{~h}$ and 1 week (1 W) post IG; Cell nuclei counterstained with HOECHST 33342 - blue fluorescence; Y2O3:Tb NPs - red fluorescence; Lectin labelled with Alexa Fluor 488 - green fluorescence 


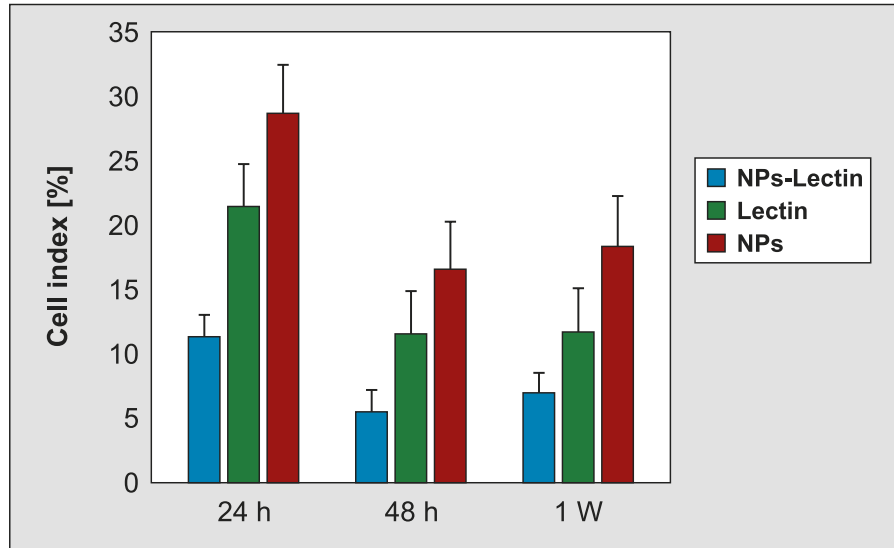

Fig. 3. Average index ( \pm SEM) of cells positive for NP-Lectin complexes, lectin and NPs alone at $24 \mathrm{~h}, 48 \mathrm{~h}$ and 1 week $(1 \mathrm{~W})$ post IG in the kidney

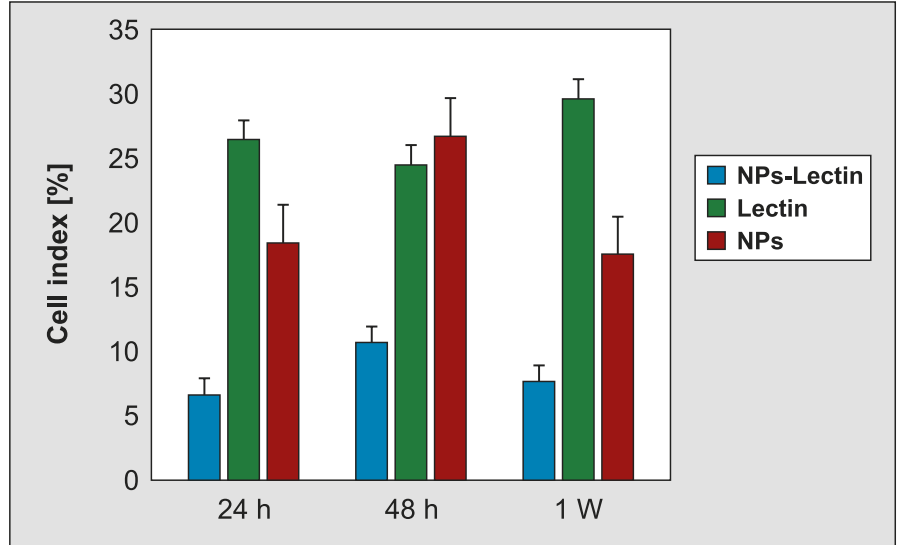

Fig. 4. Average index ( \pm SEM) of cells positive for NP-Lectin complexes, lectin and NPs alone at $24 \mathrm{~h}, 48 \mathrm{~h}$ and 1 week $(1 \mathrm{~W})$ post IG in the liver
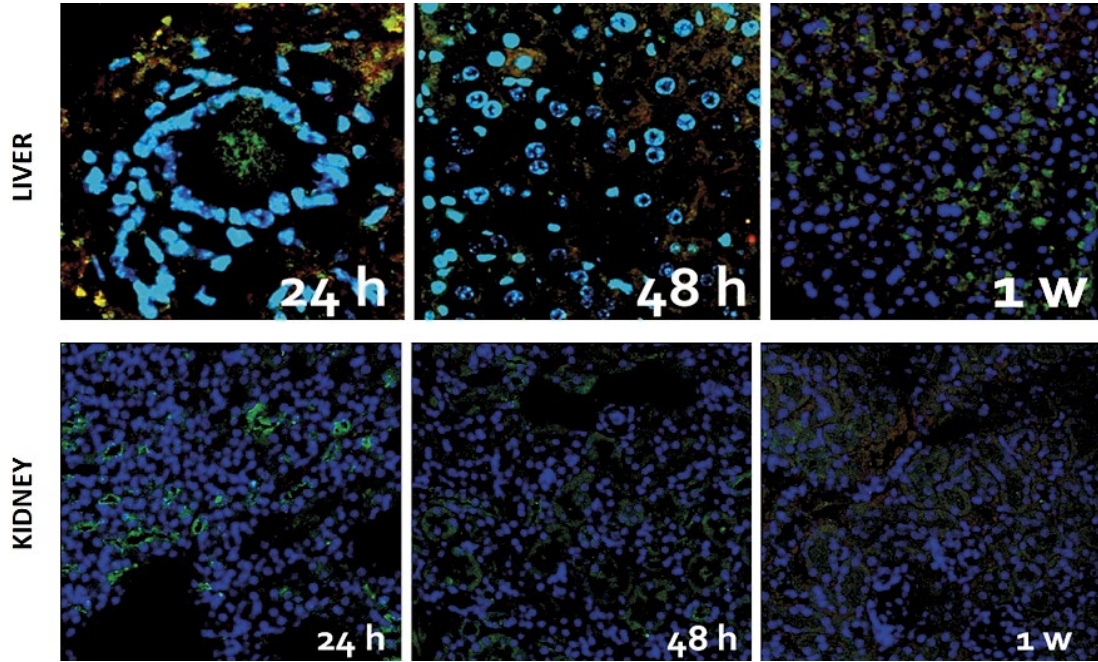

Fig. 5. Confocal image $(20 \times)$ from the liver and kidney at $24 \mathrm{~h}, 48 \mathrm{~h}$ and 1 week $(1 \mathrm{~W})$ post IG; Cell nuclei counterstained with HOECHST 33342 - blue fluorescence; Y2O3:Tb NPs - red fluorescence; lectin labelled with Alexa Fluor 488 - green fluorescence

a sharp, highly statistically significant decrease in the NP-lectin-positive cell index between 24 and $48 \mathrm{~h}$ (from over 10\% to around 5\%) (Fig. 3). Furthermore, similar results were seen for both lectin and free NPs (Fig. 3), also with highly statistical significance. Although cytometric results (Fig. 3) showed a high percentage of cells positive for free NPs, in confocal imaging (Fig. 5) red fluorescence of NPs was barely visible.

In the liver, over $5 \%$ of cells were positive for NP-lectin complexes (Fig. 4). The results also showed a high percentage of cells positive for free NPs and for lectin. None of the fluctuations observed between the time points in the percentage of cells positive for NP-lectin complexes or free lectin (Fig. 4) were statistically significant. Changes in the percentage of cells positive for free NPs (Fig. 4) were highly signifi-
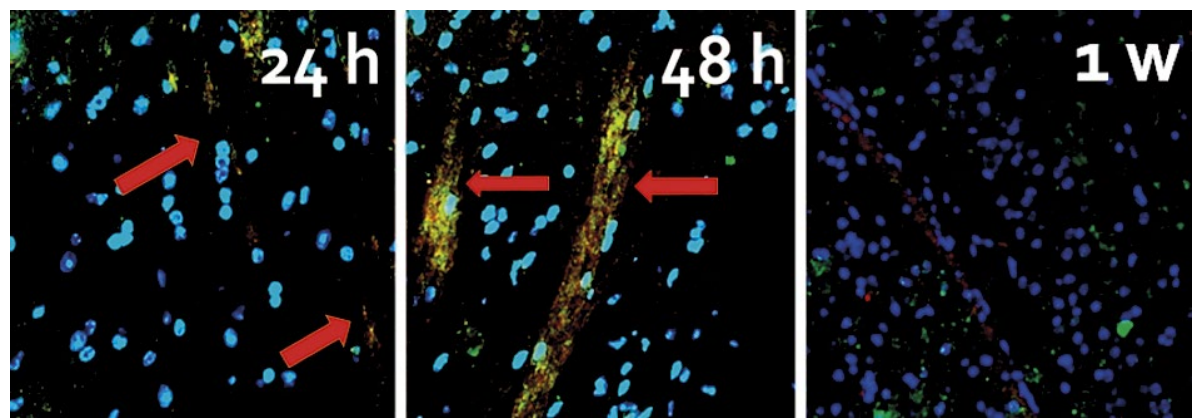

Fig. 7. Confocal image $(20 \times)$ from the brain at $24 \mathrm{~h}, 48 \mathrm{~h}$ and 1 week $(1 \mathrm{~W})$ post IG; Cell nuclei counterstained with HOECHST 33342 - blue fluorescence; Y2O3:Tb NPs - red fluorescence; Lectin labelled with Alexa Fluor 488 - green fluorescence 
out the experiment (Fig. 6). The percentage of brain cells positive for lectin (Fig. 6) statistically significantly $(\mathrm{p}<0.001)$ decreased between $24 \mathrm{~h}$ and $48 \mathrm{~h}$.

In the brain, cells positive for both NPs and lectin seemed to accumulate in a linear pattern (Fig. 7, $24 \mathrm{~h}$ and $48 \mathrm{~h}$, arrows). Contrary to scanning cytometry (Fig. 6), 1 week after IG there was no sign of colocalization between lectins and NPs observed in the brain cells at confocal imaging (Fig. 7).

The most important result of this study is a confirmation of the role of NPs as carriers of bioactive substance across an intact intestinal barrier and subsequent distribution of NP-lectin conjugates throughout the organism, including transfer through the blood-brain barrier. However, when comparing the results of this study with previous results $(1,2,5)$, a reduction in the alimentary uptake of conjugated NPs was found at $24 \mathrm{~h}$ post IG. It could be explained by either lectin blocking receptor sites from NPs or lectin attachment to the intestinal glycocalyx, which, in turn, immobilised NPs attached to lectin. At later times, the brush-border-bound fraction of lectin decreases, with NP-lectin conjugates visible only inside the cells. The persistence of the NP-related signal until 1 week after IG could be explained by previously postulated bilemediated NP recirculation between enterocytes and hepatocytes (1).

The high index of cells positive for lectin and free NPs in the liver (Fig. 4) indicate the possibility of the distribution of NP-lectin complexes via the portal vein to the liver, where some complexes undergo dissolution whereas others undergo further distribution $(1,2)$. Although changes in the cell index between the time points shown in figure 4 for NP-lectin-positive cells were statistically insignificant, they were similar to fluctuations in free NPs in the liver during distribution and redistribution patterns of NPs described previously $(1,2,5)$.

In the kidneys, the cytometric results showed a high percentage of cells positive for free NPs (Fig. 3) at all time points. However, in the confocal imaging (Fig. 5), the NP-related red fluorescence is very dim. This could be explained by the low NP content in kidney cells, similar to previous observations $(5,12)$.

Presence of NP-lectin conjugates, as well as lectin in the brain (Fig. 6 and 7), proves that conjugated NPs penetrated the blood-brain barrier. Linear colocalization pattern between NP-related and lectin-related fluorescence observed in the brain at 24 and $48 \mathrm{~h}$ (Fig. 7 , arrows) confirms the role of neuronal networks in the process of NP circulation in this organ (for reference see $5,6,11)$. One week post $\mathrm{IG}$, the colocalization between NPs and lectin observed by confocal imaging was greatly reduced, suggesting dissolution of NP-lectin complexes in the brain.

In conclusion, we found that $\mathrm{Y}_{2} \mathrm{O}_{3}: \mathrm{Tb} \mathrm{NPs}$ conjugated with lectin passed through the intestinal barrier after gastric gavage, even though the process was of lesser intensity than the one for un-conjugated NPs. Other than that, conjugation with lectin did not impair the ability of NPs to pass through blood-organ barriers, including the blood-brain barrier. In addition, after uptake and organ distribution, NP-lectin complexes were quickly dissolved, resulting in lectin deposition in tissues.

\section{References}

1. Godlewski M. M., Godlewski M.: Superradiant rare-earth doped nanocrystals in the study of persorption processes in the adult intestine, [in:] MéndezVilas A. (ed.): Current Microscopy Contributions to Advances in Science and Technology. Spain: Formatex 2012, p. 582-590

2. Godlewski M. M., Kaszewski J., Szal A., Słońska A., Domino M. A., Mijowska E., Witkowski B. S., Godlewski M.: Size of nanocrystals affects their alimentary absorption in adult mice. Med. Weter. 2014, 70, 558-563.

3. Kaszewski J., Godlewski M. M., Witkowski B. S., Stońska A., Wolska-Kornio E. Wachnicki Ł., Przybylińska H., Kozankiewicz B., Szal A., Domino M. A., Mijowska E., Godlewski M.: $\mathrm{Y}_{2} \mathrm{O}_{3}:$ Eu nanocrystals as biomarkers prepared by microwave hydrothermal method. Optical Materials 2016, 59, 157-164.

4. Kaszewski J., Witkowski B. S., Wachnicki Ł., Przybylińska H., Kozankiewicz B., Mijowska E., Godlewski M.: Reduction of $\mathrm{Tb}^{4+}$ ions in luminescent $\mathrm{Y}_{2} \mathrm{O}_{3}: \mathrm{Tb}$ nanorods prepared by microwave hydrothermal method. Journal of Rare Earths, 2016, Vol. 34, No. 8, p. 774.

5. Kietbik P., Kaszewski J., Rosowska J., Wolska E., Witkowski B. S., Gralak M. A., Gajewski Z., Godlewski M., Godlewski M. M.: Biodegradation of the $\mathrm{ZnO}:$ Eu nanoparticles in the tissues of adult mouse after alimentary application. Nanomedicine: Nanotechnology, Biology, and Medicine 2016, doi: 10.1016/j. nano.2016.11.002.

6. KielbikP., KaszewskiJ., Witkowski B. S., GajewskiZ., GralakM.A., GodlewskiM., Godlewski M. M.: Cytometric analysis of Zn-based nanoparticles for biomedical applications, [in:] Méndez-Vilas A. (ed.): Microscopy and Imaging Science. Spain: Formatex 2017, p. 89-96.

7. Lohcharoenkal W., Wang L., Chen Y. C., Rojanasakul Y.: Protein nanoparticles as drug delivery carriers for cancer therapy. BioMed Research International 2014 vol. 2014.

8. Nitta S. K., Numata K.: Biopolymer-Based Nanoparticles for Drug/Gene Delivery and Tissue Engineering. Int. J. Mol. Sci. 2013, 14, 1629-1654.

9. Shu Y., Shu D., Haque F., Guo P.: Fabrication of pRNA nanoparticles to deliver therapeutic RNAs and bioactive compounds into tumor cells. Nat. Protoc. 2013, 8 (9).

10. Siafka P. I., Okur N. U., Karavas E., Bikiaris D. N.: Surface modified multifunctional and stimuli responsive nanoparticles for drug targeting: current status and uses. Int. J. Mol. Sci. 2016, 17, 1440.

11. Słońska A., Kaszewski J., Wolska-Kornio E., Witkowski B., Wachnicki E., Mijowska E., Karakitsou V., Gajewski Z., Godlewski M., Godlewski M. M.: Luminescent properties of $\mathrm{ZrO}_{2}: \mathrm{Tb}$ nanoparticles for applications in neuroscience. Optical Materials 2016, 59, 96-102.

12. Wolska E., Kaszewski J., Kiełbik P., Grzyb J., Godlewski M. M., Godlewski M. Rare earth activated $\mathrm{ZnO}$ nanoparticles as biomarkers. Optical Materials 2014, $36,1655-1659$

13. Yatsunenko S., Kaszewski J., Grzyb J., Pelech I., Godlewski M. M., Mijowska E., Narkiewicz U., Godlewski M.: Impact of yttria stabilization on $\mathrm{Tb}^{3+}$ intrashell luminescence efficiency in zirconium dioxide nanopowders. J. Phys.: Condens. Matter 2013, (25).

14. Zhang X.-F., Liu Z.-G., Shen W., Gurunathan S.: Silver nanoparticles: nynthesis, characterization, properties, applications, and therapeutic approaches. Int. J. Mol. Sci. 2016, 17, 1534

15. Zhao Z., Li Y., Xie M.-B.: Silk fibroin-based nanoparticles for drug delivery. Int. J. Mol. Sci. 2015, 16, 4880-4903.

Corresponding author: mgr. inż. Waldemar Lipiński, Polączona 18, 03-109 Warsaw, Poland; e-mail: waldemarm.lipinski@gmail.com 